\title{
Quỹ NAFOSTED và các công bố trên hệ thống xuất bản của Nature, Science: Quả ngọt đầu mùa
}

Hồ Mạnh Toàn - Trung tâm Nghiên cứu Xã hội liên ngành ISR Trường Đại học Thành Tây, Hà Nội toan.ho@thanhtay.edu.vn

24/09/2018 11:25

https://khoahocphattrien.vn/khoa-hoc/quy-nafosted-va-cac-cong-bo-tren-he-thong-xuat-ban-cua-nature-sciencequa-ngot-dau-mua/2018092409046826p1c160.htm

\section{0 công bố đáng tự hào và đang góp phân làm nên uy tín cho NAFOSTED thời gian gân đây đều nằm trong các lĩnh vực khoa học tự nhiên và kỹ thuật và hâu hết là kết quả của hợp tác quốc tế.}

Với sứ mệnh quốc gia thúc đẩy sự tiến bộ khoa học và công nghệ, phục vụ đời sống con người, các quỹ nghiên cứu khoa học quốc gia cũng như các quỹ tài trợ khoa học tư nhân / cộng đồng luôn mong muốn những sản phẩm do mình tài trợ có thể công bố trên các ấn phẩm chất lượng cao, của những nhà xuất bản hạng nhất.

Một lý do quan trọng đó là ngoài việc nâng cao vị thế và hình ảnh của quỹ, những công bố chất lượng cao nhất còn giúp xã hội và chính phủ có thông tin để đánh giá đúng đóng góp của công việc tài trợ đối với sự tiến triển của nền khoa học.

Ở khía cạnh này, Quỹ Phát triển Khoa học và Công nghệ Quốc gia (NAFOSTED) của Việt Nam cũng không phải là ngoại lệ. Đặc biệt là trong bối cảnh của một nước đang phát triển, hiệu quả từ chi phí đầu tư cho nghiên cứu khoa học chắc chắn là một yếu tố luôn được chú ý [1].

Hiện nay, tiêu chuẩn công bố quốc tế của quỹ NAFOSTED được dựa theo hai danh mục ISI Clarivate Web of Science và Scopus - hai cơ sở dữ liệu về tạp chí khoa học hàng đầu thế giới và hầu hết các tạp chí hạng nhất đều nằm trong hai danh mục này. Tuy nhiên, trên thế giới, ngành xuất bản khoa học đã có lịch sử lâu đời với hai tạp chí đầu tiên ra mắt từ năm 1665. Chính vì thế, có những tạp chí đã trở thành tượng đài như Nature (Hệ số tác động JIF 41.577), Science (37.205), PNAS (9.661), The Lancet (45.217) hay New England Journal of Medicine (79.258). 
Bên cạnh đó, phần đông thế giới xuất bản là các hệ thống, có tính bổ trợ lẫn nhau. Do đó, bên cạnh Science thì $A A A S$ cũng có những ấn phẩm như Science Advances (11.51), Science Robotics. Tương tự, bên cạnh Nature thì NXB Nature Research còn có Nature Communications (12.353), Nature Materials (39.235), Scientific Reports (4.122), Scientific Data (5.305), v.v...

Điều khiến các tạp chí, hệ thống này trở thành những tượng đài tiên phong hoàn toàn không nằm ở con số Journal Impact Factor (Web of Science) hay CiteScore (Scopus), mà nằm ở sứ mệnh, mục tiêu và cam kết chất lượng khoa học, thông qua chất lượng biên tập và bình duyệt.

\section{Kết quả của hợp tác quốc tế trong các lĩnh vực khoa học tự nhiên và kỹ thuật}

Cho đến nay, tuy chưa nhiều, nhưng chúng ta cũng có thể liệt kê các công trình được NAFOSTED tài trợ công bố trên các hệ thống này như danh sách dưới đây:

\begin{tabular}{|c|c|c|c|c|c|c|}
\hline$\pi$ & Tên công trình & Tác giả & $\begin{array}{l}\text { Tên đơn vị } \\
\text { chủ quản của } \\
\text { tác giả người } \\
\text { Viêt }\end{array}$ & Tạp chí & $\begin{array}{l}\text { Năm } \\
\text { công } \\
\text { bố }\end{array}$ & $\begin{array}{l}\text { Chuẩn hỗ trợ } \\
\text { Open Science }\end{array}$ \\
\hline 1 & $\begin{array}{l}\text { New ZMPSTE24 } \\
\text { (FACE1) mutations } \\
\text { in patients affected } \\
\text { with restrictive } \\
\text { dermopathy or } \\
\text { related progeroid } \\
\text { syndromes and } \\
\text { mutation update }\end{array}$ & 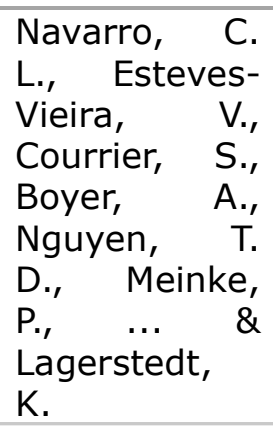 & $\begin{array}{l}\text { Viện Nghiên } \\
\text { cứu Hệ Gen } \\
\text { (Viện HL } \\
\text { KH\&CN VN), } \\
\text { Viện Về sinh } \\
\text { dịch tễ trung } \\
\text { ương }\end{array}$ & $\begin{array}{l}\text { European } \\
\text { Journal of } \\
\text { Human Genetics } \\
\text { (Nature) }\end{array}$ & 2014 & $\begin{array}{lrr}\text { Xin phép } & \text { sử } \\
\text { dụng lại } & \text { nội } \\
\text { dung bài } & \text { viết } \\
\text { thông } & \text { qua } \\
\text { RightsLink. } & \end{array}$ \\
\hline 2 & $\begin{array}{l}\text { Linking rapid } \\
\text { erosion of the } \\
\text { Mekong River delta } \\
\text { to human activities }\end{array}$ & $\begin{array}{l}\text { Anthony, E. } \\
\text { J., Brunier, } \\
\text { G., Besset, } \\
\text { M., Goichot, } \\
\text { M., } \\
\text { Dussouillez, } \\
\text { P., \& Nguyen, } \\
\text { V. L. }\end{array}$ & $\begin{array}{l}\text { Viện Địa lý } \\
\text { Tài nguyên } \\
\text { (Viện HL } \\
\text { KH\&CN VN) }\end{array}$ & $\begin{array}{l}\text { Scientific } \\
\text { Reports (Nature) }\end{array}$ & 2015 & $\begin{array}{l}\text { Creative } \\
\text { Commons } \\
\text { Attribution } 4.0 \\
\text { International } \\
\text { License }\end{array}$ \\
\hline 3 & $\begin{array}{l}\text { Large-scale } \\
\text { diversification } \\
\text { without genetic } \\
\text { isolation in } \\
\text { nematode } \\
\text { symbionts of figs }\end{array}$ & $\begin{array}{l}\text { Susoy, V., } \\
\text { Herrmann, } \\
\text { M., Kanzaki, } \\
\text { N., Kruger, } \\
\text { M., Nguyen, } \\
\text { C. } \\
\text { Rödelsperger, } \\
\text { C., } \quad \text { N. \& } \quad \text { \& } \\
\text { Sommer, R. J. }\end{array}$ & 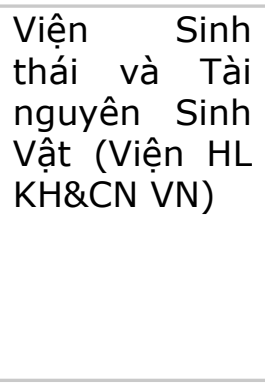 & $\begin{array}{l}\text { Science } \\
\text { Advances } \\
\text { (Science) }\end{array}$ & 2016 & $\begin{array}{l}\text { Creative } \\
\text { Commons } \\
\text { Attribution- } \\
\text { NonCommercial } \\
\text { license }\end{array}$ \\
\hline 4 & $\begin{array}{l}\text { Batrachochytrium } \\
\text { salamandrivorans is } \\
\text { the predominant } \\
\text { chytrid fungus in } \\
\text { Vietnamese } \\
\text { salamanders }\end{array}$ & $\begin{array}{l}\text { Laking, A. E., } \\
\text { Ngo, H. N., } \\
\text { Pasmans, F., } \\
\text { Martel, A., \& } \\
\text { Nguyen, T. T. }\end{array}$ & $\begin{array}{lc}\text { Bảo } & \text { Tàng } \\
\text { Thiên } & \text { nhiên } \\
\text { Việt } & \text { Nam } \\
\text { (Viện } & \text { HL } \\
\text { KH\&CN } & \text { VN) }\end{array}$ & $\begin{array}{l}\text { Scientific } \\
\text { Reports (Nature) }\end{array}$ & 2017 & $\begin{array}{l}\text { Creative } \\
\text { Commons } \\
\text { Attribution } 4.0 \\
\text { International } \\
\text { License }\end{array}$ \\
\hline 5 & $\begin{array}{l}\text { Entanglement of } \\
\text { photons in their } \\
\text { dual wave-particle }\end{array}$ & $\begin{array}{ll}\text { Rab, A. } & \text { S., } \\
\text { Polino, } & \text { E., } \\
\text { Man, Z. } & \text { X., }\end{array}$ & $\begin{array}{l}\text { Viện Vật lý } \\
\text { (Viện HL } \\
\text { KH\&CN VN) }\end{array}$ & $\begin{array}{l}\text { Nature } \\
\text { Communications } \\
\text { (Nature) }\end{array}$ & 2017 & $\begin{array}{l}\text { Creative } \\
\text { Commons } \\
\text { Attribution }\end{array}$ \\
\hline
\end{tabular}




\begin{tabular}{|c|c|c|c|c|c|c|}
\hline & nature & 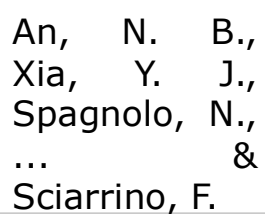 & & & & $\begin{array}{l}\text { International } \\
\text { License }\end{array}$ \\
\hline 6 & $\begin{array}{l}\text { Simultaneous } \\
\text { observation of } \\
\text { particle and wave } \\
\text { behaviors of } \\
\text { entangled photons }\end{array}$ & $\begin{array}{l}\text { Man, Z. X., } \\
\text { Xia, Y. J., \& } \\
\text { An, N. B. }\end{array}$ & $\begin{array}{l}\text { Viện Vật lý } \\
\text { (Viện HL } \\
\text { KH\&CN VN) }\end{array}$ & $\begin{array}{l}\text { Scientific } \\
\text { Reports (Nature) }\end{array}$ & 2017 & $\begin{array}{l}\text { Creative } \\
\text { Commons } \\
\text { Attribution } 4.0 \\
\text { International } \\
\text { License }\end{array}$ \\
\hline 7 & $\begin{array}{l}\text { Density functional } \\
\text { theory study of the } \\
\text { role of benzylic } \\
\text { hydrogen atoms in } \\
\text { the antioxidant } \\
\text { properties of } \\
\text { lignans }\end{array}$ & $\begin{array}{l}\text { Vo, Q. } \text { V., } \\
\text { Nam, P. C..' } \\
\text { Van Bay, M., } \\
\text { Thong, N. } \\
\text { Cuong, N. } \\
\text { \& Mechler, } \\
\text { \&.' }\end{array}$ & 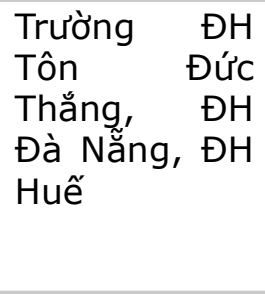 & $\begin{array}{l}\text { Scientific } \\
\text { Reports (Nature) }\end{array}$ & 2018 & $\begin{array}{l}\text { Creative } \\
\text { Commons } \\
\text { Attribution } 4.0 \\
\text { International } \\
\text { License }\end{array}$ \\
\hline 8 & $\begin{array}{lr}\text { Controlled } & \text { Defect } \\
\text { Based } & \text { Ultra } \\
\text { Broadband } & \text { Full- } \\
\text { sized Metamaterial } \\
\text { Absorber }\end{array}$ & $\begin{array}{l}\text { Tran, M. C., } \\
\text { Le, D. H.,, } \\
\text { Pham, V. H., } \\
\text { Do, H. T., Le, } \\
\text { D. T., Dang, } \\
\text { H. L., \& Vu, } \\
\text { D. L. }\end{array}$ & $\begin{array}{lr}\text { Trường } & \text { ĐH } \\
\text { SP } & H N \\
\text { Trường ĐH } \\
\text { Mỏ Địa chất } \\
\text { HN, Viện KH } \\
\text { Vật liệu và } \\
\text { Viện Vật lý } \\
\text { (Viện } \\
\text { KH\&CN Việt } \\
\text { Nam) }\end{array}$ & $\begin{array}{l}\text { Scientific } \\
\text { Reports (Nature) }\end{array}$ & 2018 & $\begin{array}{l}\text { Creative } \\
\text { Commons } \\
\text { Attribution } 4.0 \\
\text { International } \\
\text { License }\end{array}$ \\
\hline 9 & $\begin{array}{l}\text { Minor hysteresis } \\
\text { patterns with a } \\
\text { rounded/sharpened } \\
\text { reversing behavior } \\
\text { in ferromagnetic } \\
\text { multilayer }\end{array}$ & $\begin{array}{l}\text { Quach, D. T., } \\
\text { Pham, D. T., } \\
\text { Ngo, D. T., } \\
\text { Phan, T. L., } \\
\text { Park, S. Y., } \\
\text { Lee, S. H., \& } \\
\text { Kim, D. H. }\end{array}$ & 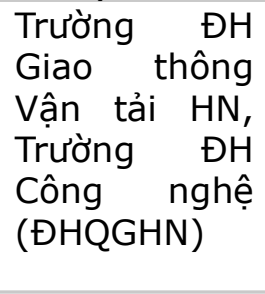 & $\begin{array}{l}\text { Scientific } \\
\text { Reports (Nature) }\end{array}$ & 2018 & $\begin{array}{l}\text { Creative } \\
\text { Commons } \\
\text { Attribution } 4.0 \\
\text { International } \\
\text { License }\end{array}$ \\
\hline 10 & $\begin{array}{l}\text { Ultra- } \\
\text { subwavelength } \\
\text { thickness for } \\
\text { dual/triple-band } \\
\text { metamaterial } \\
\text { absorber at very } \\
\text { low frequency }\end{array}$ & $\begin{array}{l}\text { Khuyen, }{ }^{\text {B. }} \\
\text { X., Tung, B. } \\
\text { S., Kim, Y. J., } \\
\text { Hwang, J. S., } \\
\text { Kim, K. W., } \\
\text { Rhee, J. Y., ... } \\
\text { \& Lee, Y. }\end{array}$ & $\begin{array}{l}\text { Viện KH Vật } \\
\text { liệu (Viện HL } \\
\text { KH\&CN VN) }\end{array}$ & $\begin{array}{l}\text { Scientific } \\
\text { Reports (Nature) }\end{array}$ & 2018 & $\begin{array}{l}\text { Creative } \\
\text { Commons } \\
\text { Attribution } 4.0 \\
\text { International } \\
\text { License }\end{array}$ \\
\hline
\end{tabular}

Trong đó, Nature Communications là tạp chí có hệ số tác động JIF cao nhất (12.353) và Scientific Reports là địa chỉ quen thuộc nhất (7/10 công bố).

Hầu hết các nghiên cứu đều là hợp tác quốc tế và chỉ có duy nhất nghiên cứu "Controlled Defect Based Ultra Broadband Full-sized Metamaterial Absorber" được tài trợ toàn phần bởi NAFOSTED và cũng là nghiên cứu duy nhất không có tác giả quốc tế. Các nghiên cứu còn lại đều được hỗ trợ hoặc tài trợ một phần bởi các tổ chức quốc tế.

Cả 10 tác phẩm đều được xuất bản Open-Access với 9/10 tác phẩm đạt chuẩn Creative Commons Attribution 4.0 International License (chuẩn về dữ liệu mở mới nhất hiện nay). Hơn thế nữa, nghiên cứu "Entanglement of photons in their dual wave-particle nature" trên tạp chí Nature Communications còn đi một bước xa hơn; các tác giả đã công khai dữ liệu và báo cáo phản biện. Điều này cho thấy quỹ 
NAFOSTED và các tác giả công trình công bố cũng như các đơn vị chủ quản đang có hướng đi đúng đắn, phù hợp với xu hướng khoa học mở của khoa học thế giới hiện nay $[2,3]$.

Tuy nhiên, có thể thấy thực tế 10 công bố đáng tự hào và đang góp phần gây dựng truyền thống NAFOSTED liệt kê ở trên đều nằm trong các lĩnh vực khoa học tự nhiên và kỹ thuật (vật lý, sinh học, địa lý). Đây đều là những lĩnh vực chuyên môn có truyền thống lâu đời và có lịch sử hợp tác quốc tế. Một điểm lưu ý khác là các công trình công bố chủ yếu mới chỉ xuất hiện trong khoảng 2 năm gần đây (7/10 công trình công bố trong 2017 và 2018). Chúng ta cùng hy vọng trong tương lai sẽ có nhiều công bố hơn từ các tác giả người Việt, đặc biệt là các nhóm hoàn toàn Việt Nam có bài công bố trên hệ thống xuất bản của Nature và Science do NAFOSTED tài trợ.

\section{Tham khảo:}

[1] Vuong, Q. H. (2018). The (ir)rational consideration of the cost of science in transition economies. Nature Human Behaviour, 2(1), 5, DOI:10.1038/s41562-017-0281-4. < https://www.nature.com /articles/s41562-017-0281-4 >

[2] Wilkinson, M. D., Dumontier, M., Aalbersberg, I. J., Appleton, G., Axton, M., Baak, A., ... \& Bouwman, J. (2016). The FAIR guiding principles for scientific data management and stewardship. Scientific Data, 3, 160018, DOI:10.1038/sdata.2016.18. < https://www.nature.com/articles /sdata201618 >

[3] Editorial. (2017). Open for business. Scientific Data, 4, 170058, DOI:10.1038/sdata.2017.58. < https://www.nature.com/articles/sdata201758 >

3 comments on PubPeer (by: Peter R. Oxley, Bunomys Andrewsi, Carl Taswell)

Hồ Mạnh Toàn - Trung tâm Nghiên cứu Xã hội liên ngành ISR Trường Đại học Thành Tây, Hà Nội toan.ho@thanhtay.edu.vn 


\section{Tài liệu tham khảo:}

[1] Vuong, Q. H. (2018). The (ir)rational consideration of the cost of science in transition economies. Nature Human Behaviour, 2(1), 5.

[2] Wilkinson, M. D., Dumontier, M., Aalbersberg, I. J., Appleton, G., Axton, M., Baak, A., ... \& Bouwman, J. (2016). The FAIR guiding principles for scientific data management and stewardship. Scientific Data, 3, 160018.

[3] Editorial. (2017). Open for business. Scientific Data, 4(1), 170058. 